\title{
Interaction between electronic structure and strain in Bi nanolines on $\mathrm{Si}(001)$
}

\author{
J.H.G. Owen ${ }^{\mathrm{a}, \mathrm{b}, *, 1}$, K.Miki ${ }^{\mathrm{a}, \mathrm{b}}$ and D.R. Bowler ${ }^{\mathrm{c}, 2}$ \\ ${ }^{a}$ Nanotechnology Research Institute (NRI), National Institute of Advanced \\ Industrial Science and Technology (AIST), 1-1-1 Higashi, Tsukuba. Ibaraki \\ 305-8562, Japan \\ ${ }^{\mathrm{b}}$ Nanomaterials Laboratory (NML), National Research Institute for Materials \\ Science (NIMS) Sengen 1-2-1, Tsukuba. Ibaraki 305-0047, Japan, \\ ${ }^{\mathrm{c}}$ Department of Physics and Astronomy, University College London, Gower Street, \\ London WC1E 6BT, UK
}

\begin{abstract}
Heteroepitaxial strain can be a controlling factor in the lateral dimensions of 1-D nanostructures. Bi nanolines on $\mathrm{Si}(001)$ have an atomic structure which involves a large sub-surface reconstruction, resulting in a strong elastic coupling to the surrounding silicon. We present variable-bias STM and first principles electronic structure calculations of the $\mathrm{Bi}$ nanolines, which investigates this interaction. We show that the strain associated with the nanolines affects the atomic and electronic structure of at least two neighbouring Si dimers, and identify the mechanism behind this. We also present partial charge densities (projected by energy) for the nanoline with clean and hydrogenated surroundings and contrast it to the clean $\mathrm{Si}(001)$ surface.
\end{abstract}

Key words:

\footnotetext{
* Corresponding author

Email addresses: james.owen@materials.ox.ac.uk (J.H.G. Owen), miki.kazushi@aist.go.jp (K.Miki), david.bowler@ucl.ac.uk (D.R. Bowler).

URL: http://www.cmmp.ucl.ac.uk/ drb/research.html (D.R. Bowler).

1 Present address : Dept. of Materials, Oxford University, Parks Rd,Oxford, OX1 $3 \mathrm{PH}, \mathrm{UK}$

2 Also at: London Centre for Nanotechnology, Department of Physics and Astronomy, Gower Street, London WC1E 6BT, UK
} 


\section{Introduction}

Nanometer-scale electronic technologies require not only the formation of nanoscale devices, but also nanoscale interconnections between the devices. At present, nanoscale structures may be made via at least three different approaches, all of which are likely to used in any scheme: top-down lithographic methods, such as e-beam lithography and AFM lithography; bottom-up methods, which involve positioning of ex situ "prefabricated" structures such as conducting molecules, semiconductor nanowires[1] and carbon nanotubes[2] using, e.g., scanning probes or micro-fluidics; or by in situ self-assembly, as in semiconductor quantum dots, or silicide nanowires[3]. We are pursuing a hybrid self-assembly-based fabrication route, whereby a Bi nanoline[4,5] is used as a nanowire or as a template for nanowires of other metals. These nanolines are very long - over $400 \mathrm{~nm}$ in some cases - and straight: a kink in a nanoline has never been seen. Unlike silicide nanowires[3], their width is constant, occupying the space of 4 dimers $(1.5 \mathrm{~nm})$ in the $\operatorname{Si}(001)$ surface. However, variable-bias STM of the nanolines indicates that they have a band gap which is larger than the surrounding surface, and suggests that they are not conductive[4,5].

Recently, we used a combination of atomistic structure calculations and experiments to identify the structure of the nanoline[6,7] (which we have called the Haiku structure). An example of the Bi nanoline on a clean $\mathrm{Si}(001)$ surface is shown in Fig. 1, with the Haiku structure shown in the inset. It has a complex reconstruction in the silicon substrate reaching down five layers below the surface, though the surrounding surface is not reconstructed. The depth of the reconstruction suggests that the nanoline may be coupled strongly to its surroundings. Knowledge of the nanoline structure allows us for the first time to make detailed calculations of the electronic structure of the nanoline, which bear out the observations made in STM. The calculations also provide an explanation for the additional features that we have observed in recent atomic-resolution variable-bias STM of the Bi nanoline on the clean $\mathrm{Si}(001)$ surface at room temperature.

In this letter, we present high resolution, variable bias images of a Bi nanoline, and DFT calculations which explore the electronic structure of the $\mathrm{Bi}$ nanoline. In the next section, we discuss details of the experimental and theoretical techniques used. We then present the atomic-resolution images of the Bi-exposed $\mathrm{Si}(001)$ surface and interpret them in light of the various calculations. 


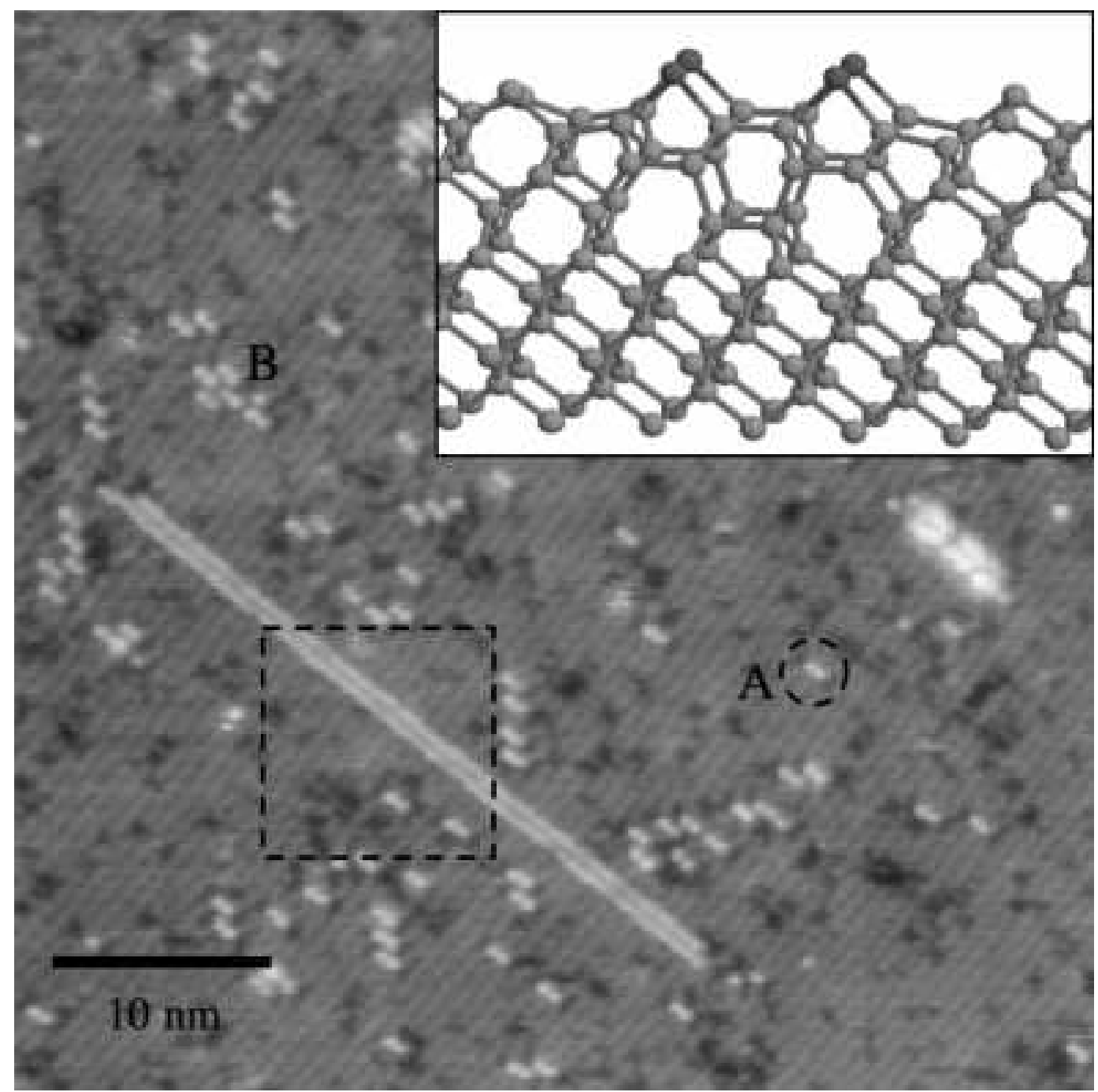

Fig. 1. A $50 \mathrm{~nm} \times 50 \mathrm{~nm} \mathrm{STM}$ image of a small section of the $\mathrm{Si}(001)$ surface containing a short nanoline, and numerous small double-dot features (A). Note that some of the double-dot features have formed small clusters in a diagonal or $\mathrm{c}(4 \times 4)$ arrangement $(\mathrm{B})$. The dotted box marks the area shown in Fig. 2. Inset: The Haiku structure for the Bi nanoline[6]. A pair of subsurface 7-membered rings of Si form the basis of this unusual reconstruction.

\section{Experimental and Theoretical Methods}

The $\mathrm{Si}(001)$ substrate was cleaned using a standard process[8] before being transferred into vacuum. The Si surface was prepared by flashing repeatedly to $1100^{\circ} \mathrm{C}$ for a few seconds, until there was only a small pressure rise. The clean surface was checked with STM before Bi deposition began. Bi was evaporated from an effusion cell, a typical dose being $\mathrm{Bi}$ at $470^{\circ} \mathrm{C}$ for 10 mins. STM images were taken at the deposition temperature between $570-600^{\circ} \mathrm{C}$, and at room temperature, using a JEOL 4500 XT UHV STM. The standard recipe for the formation of the $\mathrm{Bi}$ nanolines is to anneal the $\mathrm{Si}(001)$ surface close 
to $600^{\circ} \mathrm{C}$, under a $\mathrm{Bi}$ flux of around $1 \mathrm{ML} / \mathrm{min}$.[4]. In this case, the surface was annealed at a slightly lower temperature, around $570^{\circ} \mathrm{C}$, and quenched to $300^{\circ} \mathrm{C}$ as soon as the presence of nanolines had been confirmed. In this way, we hoped to capture Bi nanolines while still growing, and hence make some observations which would lead to a likely nucleation mechanism. To minimise surface contamination, the sample was held at $300^{\circ} \mathrm{C}$ while the $\mathrm{Bi}$ cell was cooled and the chamber pressure returned to its base level, before the sample was cooled to room temperature.

The density functional theory (DFT) calculations were performed in the Generalised Gradient Approximation (GGA) $[9,10]$ using the VASP code[11], with ultrasoft pseudopotentials, a plane wave cutoff of $150 \mathrm{eV}$ (sufficient for energy difference convergence) and a Monkhurst-Pack k-point mesh with $4 \times 4 \times 1$ points. The unit cell used contained a single Bi nanoline, and had ten layers of $\mathrm{Si}$, with twenty atoms in each layer (forming a single dimer row ten dimers long with the $\mathrm{p}(2 \times 2)$ reconstruction $)$ with the bottom two layers constrained to remain fixed and dangling bonds terminated in hydrogen. This unit cell was of sufficient size to for convergence of energy with cell depth, and long enough that the end $\mathrm{Si}$ dimers were representative of the clean, undistorted surface.

\section{$3 \quad$ Electronic structure of $\mathrm{Bi}$ nanoline}

Figure 1 shows a typical image from our STM experiments. Note that the $\mathrm{Si}(001)$ surface is clean, and that the image was taken at room temperature (in contrast to all previous experiments, where either the substrate was hydrogenated or the temperature was $\sim 570^{\circ} \mathrm{C}$ ). There are two key features to note: first, the Bi nanoline, which is the double line extending diagonally across the image from top left to bottom right; second, the features (marked 'A' in Fig.1) which appear as a double dot, and which are scattered across the surface; note that these features tend to align diagonally with each other, even forming a local $\mathrm{c}(4 \times 4)$ pattern in places (marked 'B'). In this paper, we will concentrate on the electronic structure of the nanoline. We are actively investigating a possible structure for the double-dot features, which may be a precursor to the nanoline, and we shall present our results in future work.

In order to probe more closely the physical and electronic structure of the nanoline, a series of atomic-resolution variable bias STM images (in the area shown by the dotted box in Fig. 1) were taken and are shown in Fig. 2. The STM bias voltages are given in the figure caption. Filled-states images range from $-2.0 \mathrm{~V}$ down to $-0.3 \mathrm{~V}$. An empty-states image at a bias voltage of $+1.5 \mathrm{~V}$ is also presented. As has been previously reported[4,5], the Bi nanoline appears bright at large bias voltages of either sign, but as the bias voltage is reduced, the contrast of the nanoline reduces so that at around $-0.8 \mathrm{~V}$, the 

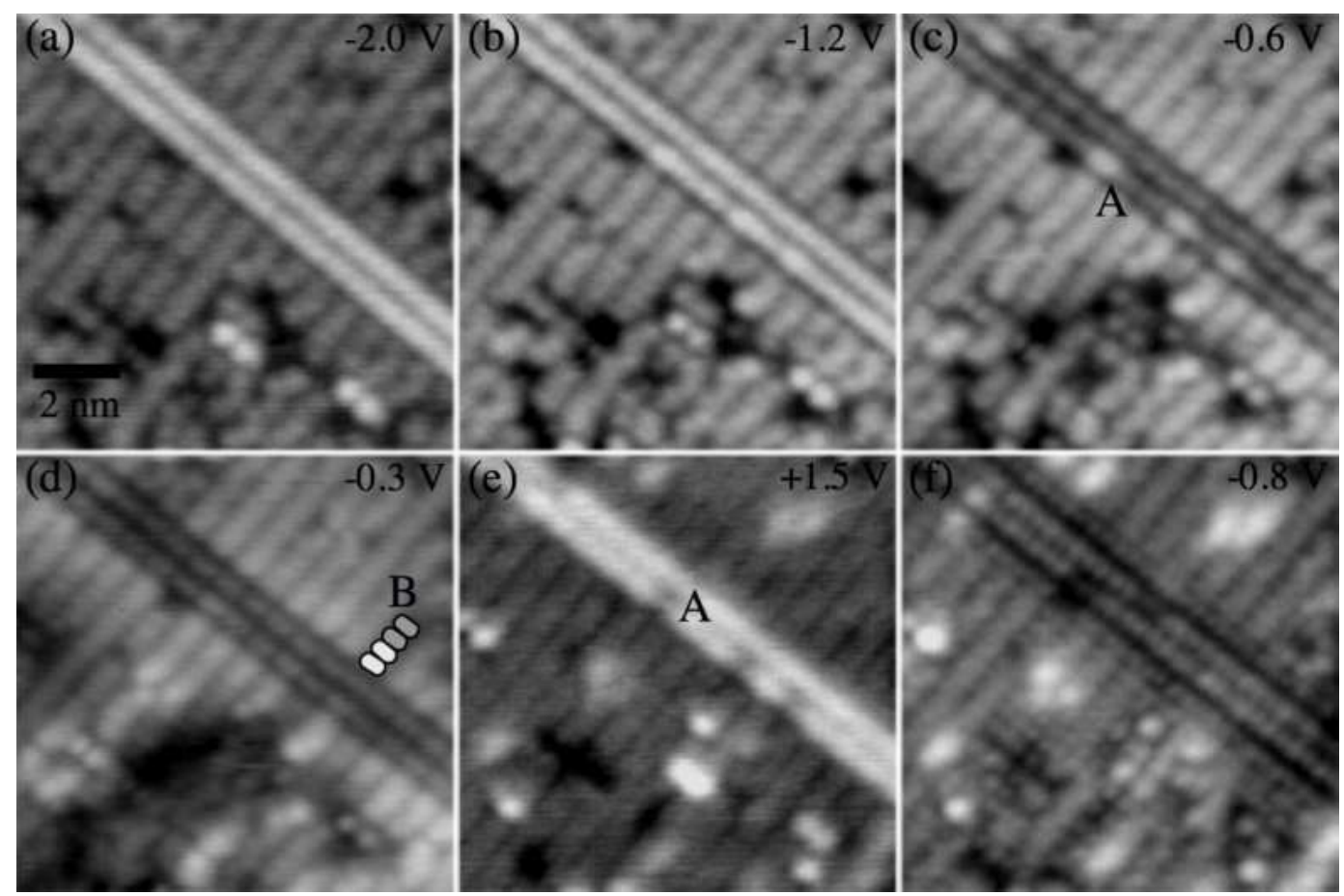

Fig. $2.10 \mathrm{~nm} \times 10 \mathrm{~nm}$ insets from the area shown in the dotted black box in Fig. 1 . The sample bias voltages used are $-2.0 \mathrm{~V},-1.2 \mathrm{~V},-0.6 \mathrm{~V},-0.3 \mathrm{~V},+1.5 \mathrm{~V}$ and $-0.8 \mathrm{~V}$, in (a-f) respectively. As the sample bias is reduced, between (a) and (d), the nanoline changes contrast from light to dark relative to the surrounding $\mathrm{Si}(001)$. Over this range, some of the dimers in the nanoline (marked A in (c) and (e)) exhibit a different voltage contrast. At very low biases, around $-0.3 \mathrm{~V}$, an enhancement of the dimers around the nanoline, similar to that seen around a missing dimer defect in clean $\operatorname{Si}(001)$ [13], is seen. This is visible (and marked schematically as B) in (d). In (f), the resolution is sufficient to see that the corrugation of the Si dimers closest to the nanoline is increased, suggesting a greater separation, and hence tensile strain.

relative contrast of the nanoline and the Si substrate is the same, and at lower bias voltages, the nanoline becomes dark relative to the clean Si surface. By contrast, images of the Bi nanoline on the $\mathrm{H}: \mathrm{Si}(001)$ surface[12] always show the nanoline as a bright feature.

At very low biases, as in Fig. 2(d), substrate dimers either side of the nanoline become enhanced, i.e. appear to be brighter than the rest of the Si dimers, out to a distance of at least two dimers. (This is shown schematically in (d), by the lighter and darker rectangles.) This phenomenon of enhancement of neighbouring dimers at low bias voltages has been seen previously for single missing dimer defects (1DV) on $\mathrm{Si}(001)[13]$. In that case, the enhancement was explained by the distortion of these dimers away from their normal structure by the strain field of the $1 \mathrm{DV}$, resulting in a local change of the electronic structure, and the top-most occupied states being moved higher in energy. The tensile strain around the $\mathrm{Bi}$ nanoline is expected to be the cause of the enhancement seen here; this enhancement is modelled below. In Fig. 2(f) (which 
shows extremely high resolution), tensile strain near the Bi nanoline is seen directly: the darkening between the first and second Si dimers is more pronounced than between the second and third Si dimers, indicating an increase in separation between these dimers. In our DFT modelling, the dimer-dimer spacing close to the nanoline is increased by $3 \%$, a significant strain.

Another feature visible in the images is a change of contrast between Bi dimers in the nanoline. While at elevated temperatures the Bi nanoline always appears uniform, at room temperature, higher resolutions may be achieved, and some subtle details resolved. Some dimers in the Bi nanoline in Fig. 2(a) appear different to the rest of the line (they seem to have a dark boundary around them); these separated dimers correspond to brighter dimers on the otherwise dark nanoline in the lower bias images (especially Fig 2(c)) and darker patches (marked as 'A') in the empty states image shown in Fig. 2(e). The reason for these features have not been determined. However, they are not seen in elevated-temperature images of the clean $\mathrm{Bi}$ nanoline, even at the same bias voltages, while similar dark patches were observed during experiments with adsorbed hydrogen and oxygen[12]. It is therefore quite possible that they are the result of contamination by ambient water or hydrogen while cooling down to room temperature.

We have calculated the electronic structure of the Haiku model for the $\mathrm{Bi}$ nanoline using DFT, as described above. In order to understand the voltage contrast seen in STM, we have projected out the charge density associated with various states within certain energies of the Fermi level. We show the partial charge densities associated with all states within $0.2 \mathrm{eV}, 0.6 \mathrm{eV}$ and $1.0 \mathrm{eV}$ of the Fermi energy in Figure 3 along with the complete charge density, for three systems: (a) the clean $\mathrm{Si}(001)$ surface; (b) the Haiku model of the Bi nanoline; and (c) the Haiku model with a hydrogenated $\mathrm{Si}(001)$ surface. For the last, the first image is missing as the first states occur $0.55 \mathrm{eV}$ below the Fermi level (a side-on view of the Haiku structure is shown in its place). The partial densities are shown in a plane passing through a Si dimer atom. As the results are for a statically buckled Si(001) surface, alternate dimers are "up" and "down" (except on the hydrogenated surface, where the buckling does not occur). Only the up atoms appear bright, resulting in an appearance of alternate missing dimers; the difference is most clearly seen by comparison of the electron charge densities with the structural model of the Haiku shown in the top right-hand corner of Fig. 3.

The partial densities are important for the comparison with experiment, since the states close to the Fermi level will contribute strongly to the STM images at low biases. Localisation of a state near the Fermi level in the area around particular atoms or bonds (in comparison to bulk or clean $\mathrm{Si}$ ) is indicative of a local strain, because strain forces structures away from their equilibrium state, and hence minimum energy configuration. We first identified this connection 
(a): Clean $\mathrm{Si}(001)$

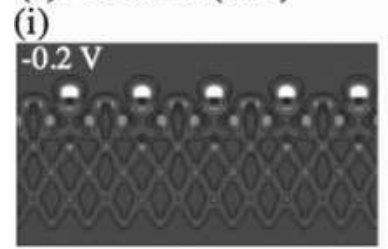

(ii)

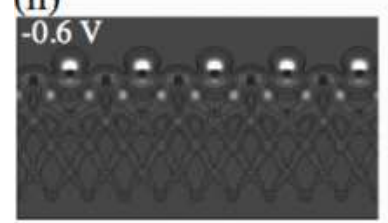

(iii)

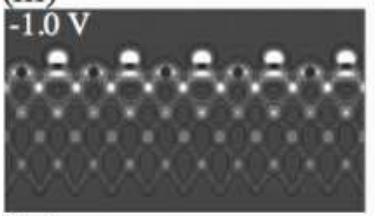

(iv)

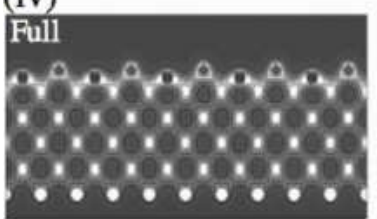

(b): Nanoline on (Si001)
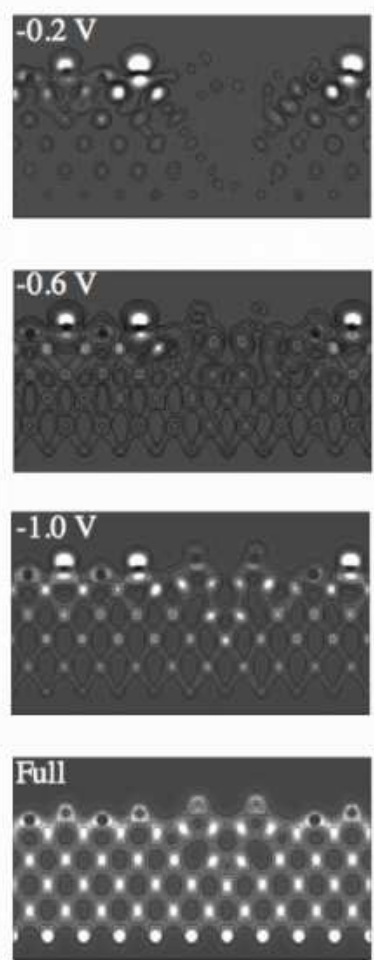

(c): Nanoline on $\mathrm{Si}(001): \mathrm{H}$
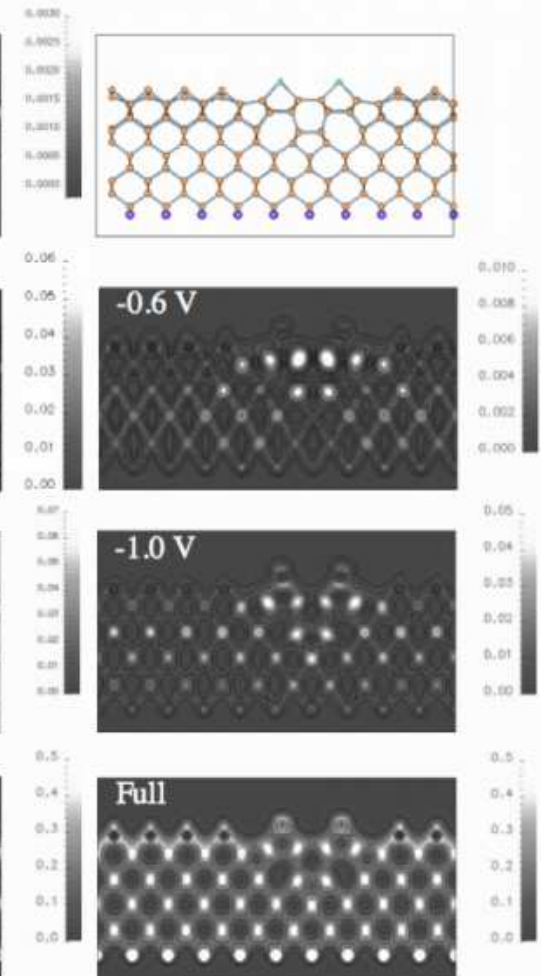

Fig. 3. Contour plots of charge density for the states (i) $0.2 \mathrm{eV}$, (ii) $0.6 \mathrm{eV}$ and (iii) $1.0 \mathrm{eV}$ below $\mathrm{E}_{F}$, and (iv) the total charge density for: (a) the clean $\mathrm{Si}(001)$ surface (first column); (b) the Haiku structure on the clean surface (second column), and (c) the hydrogenated surface (third column). The $0.2 \mathrm{eV}$ image for the hydrogenated surface has been replaced with a ball-and-stick model of the Haiku structure, as there are no states within $0.2 \mathrm{eV}$ of $\mathrm{E}_{F}$. See text for a full discussion.

between strain and STM contrast (or states moved higher in energy) in the case of the single missing dimer defect on $\mathrm{Si}(001)[13]$, and we will use it here to elucidate the substrate distortions around the Haiku structure. In the case of the clean $\mathrm{Si}(001)$ surface (first column of Fig. 3), the states near the Fermi level $(\mathrm{a}, \mathrm{i})$ are mainly localised on the surface dimers, and the density fades away beneath the surface layer. This is as expected, since the dimerisation of the $\mathrm{Si}(001)$ surface is associated both with dangling bonds (at the surface) and with strain (sub-surface). By contrast, the states near the Fermi level for the Bi nanoline on clean $\operatorname{Si}(001)$ (b,i) are reduced in density below the $\mathrm{Si}$ dimers, and are entirely excluded from the Haiku structure and substructure. Instead, the two Si dimers immediately to the side of the Haiku show a greatly enhanced charge density in the states within $0.2 \mathrm{eV}$ of the Fermi level, both by comparison with their neighbours (the left-most dimers in (b,i)) and with the clean $\mathrm{Si}(001)$ surface $(a, i)$, which is seen in STM as the brightening of the dimers either side of the Haiku at very low biases (Fig. 2(d) in particular). The localisation of these low-energy states indicates that the substructure of the Haiku, far from being a strained structure is more relaxed than the layers 
beneath a clean $\mathrm{Si}(001)$ surface. Furthermore, it demonstrates that the strain induced by the $\mathrm{Bi}$ nanoline is concentrated on the $\mathrm{Si}$ dimers either side of the Haiku structure, and on the second-to-third layer bonds beneath the dimers.

The electronic structure of the Bi nanoline with a H-terminated $\mathrm{Si}(001)$ surface is shown in Fig. 3(c). The major effect is the removal of the surface states associated with the Si dimer $\pi$-bonds, and the removal of the buckling on the surface. The Si atoms immediately beneath the Bi nanoline now show an increased charge density relative to the neighbouring $\mathrm{Si}$ atoms, which is clearly visible in the $0.6 \mathrm{eV}$ and $1.0 \mathrm{eV}$ images (c,ii \& iii), indicating that the region below the $\mathrm{Bi}$ nanoline is more strained than bulk Si. Furthermore, enhancement can be seen in layers as deep as the sixth layer, indicating the deep strain caused by the presence of the Bi nanoline. This difference in the electronic states suggests that the contrast seen with the clean surface is due to the interaction of the buckled dimers and the nanoline (and in particular the "up" atom of a buckled dimer) which is removed with a hydrogenated surface.

The lack of any states on the Bi near the Fermi level is clearly the cause of the darkening of the nanoline relative to the $\mathrm{Si}(001)$ surface seen in STM at low voltages. Indeed, the first states seen on the nanoline lie over $0.5 \mathrm{eV}$ away from the Fermi level (which agrees qualitatively with the bias voltage of $0.8 \mathrm{~V}$ where parity of appearance between the nanoline and the substrate occurs). In the full charge densities(Fig. 3(iv)), the density of states associated with the Bi dimers, combined with their higher physical height, explains their relative brightness in STM at higher bias voltages. In the case of the hydrogenated surface, the $\mathrm{Bi}$ dimers have a similar charge density as in the clean surface, but in this case, the Si $\pi$-bonds have been eliminated, so the Bi dimer remains bright in STM at all biases[12]. The large density associated with the nanoline in the $0.6 \mathrm{eV}(\mathrm{c}, \mathrm{ii})$ and $1.0 \mathrm{eV}(\mathrm{c}, \mathrm{iii})$ images underline the mechanism behind the brightening of the $\mathrm{Bi}$ nanoline.

\section{Conclusions}

We have presented high resolution room temperature STM images of Bi nanolines on a clean $\mathrm{Si}(001)$ surface for the first time. These images allowed us to probe the electronic structure of the nanoline and the surrounding $\operatorname{Si}(001)$ surface, and, by extrapolation, the localised strain associated with the Haiku. We found that the two Si dimers neighbouring the nanoline show enhancement at low bias voltages, and we confirmed that the nanoline is darker than the surrounding surface for biases of less than $0.8 \mathrm{~V}$. We have also presented first principles electronic structure calculations of the Haiku structure with both a clean and a hydrogenated $\operatorname{Si}(001)$ surface, and we have explained the voltage 
contrast of the nanolines using these calculations.

The localisation of the states near the Fermi level seen in the projected charge densities suggests that the Si atoms immediately surrounding the Haiku structure are strained (in part at least due to the interaction with the buckled Si dimers), while the Si substructure immediately beneath the Haiku itself is somewhat relaxed (though the hydrogenated surface plots suggest that it is slightly strained relative to perfect bulk $\mathrm{Si}$ ). This goes some way to explaining the stability of the subsurface 5-7-5 ring structures, which serve as a highly effective relief mechanism for the epitaxial stress exerted by adsorbed $\mathrm{Bi}$. The absence of states close to the Fermi level suggests that the nanolines are likely to block surface conduction perpendicular to the nanoline, and are not likely to act as a nanowire.

The Bi nanolines are clearly not suitable for conduction on their own. However, as we have shown before[12], hydrogen will adsorb preferentially on the $\mathrm{Si}(001)$ surface and not on the $\mathrm{Bi}$, giving a natural, automatic masking technique. This should allow us to adsorb metals on the nanolines, creating exceptionally high quality nanowires. This research is under way, and will be presented in future work.

\section{Acknowledgements}

DRB thanks the Royal Society for funding through a University Research Fellowship. Calculations were performed at the HiPerSPACE Centre at UCL (JREI grant JR98UCGI). JHGO was supported by the Japanese Science and Technology Agency (JST) as an STA Fellow. This study was performed through Special Coordination Funds of the Ministry of Education, Culture, Sports, Science and Technology of the Japanese Government (Research Project on active atom-wire interconnects). We would like to thank Bill McMahon for bringing to our attention many of the examples of 5-7-5 structures, and for sharing unpublished STM data.

\section{References}

[1] Y.Huang, X.Duan, Y.Cui, L.J.Lauhon, K.-H.Kim, C.M.Lieber, Science 294 (2001) 1313.

[2] A.Bachtold, P.Hadley, T.Nakanishi, C.Dekker, Science 294 (2001) 1317.

[3] Y. Chen, D.A.A.Ohlberg, G. Medeiros-Ribeiro, Y.A.Chang, R.S.Williams, Appl. Phys. Lett. 76 (2000) 4004. 
[4] K.Miki, J.H.G.Owen, D.R.Bowler, G.A.D.Briggs, K.Sakamoto, Surf. Sci. 421 (1999) 397.

[5] K.Miki, D.R.Bowler, J.H.G.Owen, G.A.D.Briggs, K.Sakamoto, Phys. Rev. B 59 (1999) 14868.

[6] J.H.G.Owen, K.Miki, H.Koh, H.W.Yeom, D.R.Bowler, Phys. Rev. Lett. 88 (2002) 226104.

[7] D.R.Bowler, J.H.G.Owen, J. Phys.:Condens. Matter 14 (2002) 6761.

[8] K.Miki, K.Sakamoto, T. Sakamoto, Surf. Sci. 406 (1998) 312.

[9] Y.Wang, J.P.Perdew, Phys. Rev. B 44 (1991) 13298.

[10] J.P.Perdew, J.A.Chevary, S.H.Vosko, K.A.Jackson, M.R.Pederson, D.J.Singh, C.Fiolhais, Phys. Rev. B 46 (1992) 6671.

[11] G.Kresse, J.Furthmüller, Comp. Mat. Sci. 6 (1996) 15.

[12] J.H.G.Owen, D.R.Bowler, K.Miki, Surf. Sci. Lett. 499 (2002) L124.

[13] J.H.G.Owen, D.R.Bowler, C.M.Goringe, K.Miki, G.A.D.Briggs, Surf. Sci. Lett. 341 (1995) L1042. 